\title{
Piezoelectric oxide semiconductor field effect transistor touch sensing devices
}

\author{
Ravinder S. Dahiya, ${ }^{1, a)}$ Giorgio Metta, $\left.{ }^{1, b}\right)$ Maurizio Valle, ${ }^{2}$ Andrea Adami, ${ }^{3}$ and \\ Leandro Lorenzelli ${ }^{3}$ \\ ${ }^{1}$ RBCS, Italian Institute of Technology, Genoa 16163, Italy \\ ${ }^{2}$ DIBE, University of Genoa, 16145, Italy \\ ${ }^{3}$ Bio-MEMS Group, Fondazione Bruno Kesseler, Povo, Trento 38050, Italy
}

(Received 26 March 2009; accepted 26 June 2009; published online 21 July 2009)

\begin{abstract}
This work presents piezoelectric oxide semiconductor field effect transistor (POSFET) based touch sensing devices. These devices are fabricated by spin coating thin $(\sim 2.5 \mu \mathrm{m})$ piezoelectric polymer film directly on to the gate area of metal oxide semiconductor (MOS) transistor. The polymer film is processed in situ and challenging issues such as in situ poling of piezoelectric polymer film, without damaging or altering the characteristics of underlying MOS devices, are successfully dealt with. The POSFET device represents an integral "sensotronic" unit comprising of transducer and the transistor-thereby sensing as well as conditioning (and processing) the touch signal at "same site." (c) 2009 American Institute of Physics. [DOI: 10.1063/1.3184579]
\end{abstract}

Touch sensing plays an important role in application domains such as robotics, electrotextiles, and medical prosthesis as it facilitates the detection and measurement of interaction parameters of real world objects. Over the years, touch sensing technology has improved and many new touch sensors, using different materials and transduction methods, namely, resistive/piezoresistive, capacitive, optical, magnetic, piezoelectric, etc., have been developed. ${ }^{1}$ Most of these sensors are big in size and hence unsuitable for application areas such as robotic fingertips, where large number of sensors are needed in a limited space. Miniaturized touch sensors using microelectromechanical system approach have also been developed. ${ }^{2}$ However, inherent fragile nature of such sensors limits their usage to applications involving small contact forces only. Mechanically flexible touch sensors using organic field effect transistor (FET) have also been

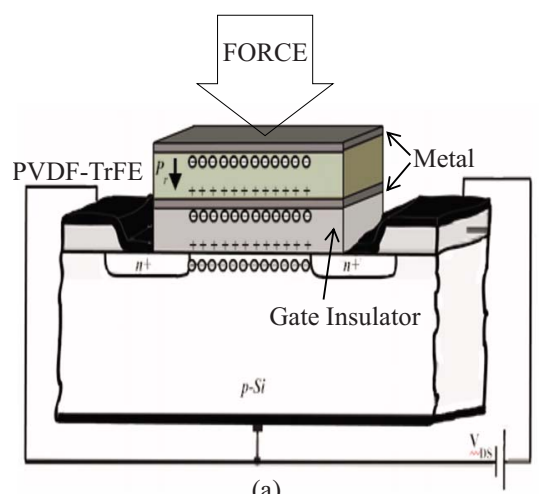

(a)

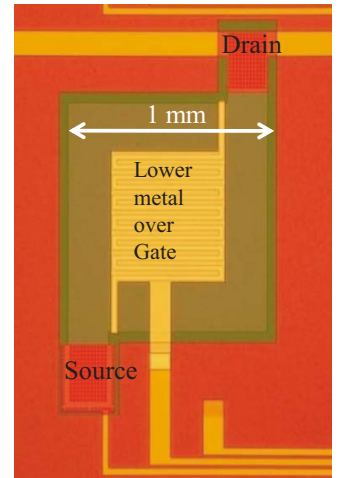

(c)

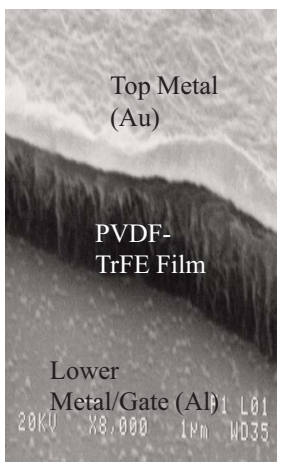

(b)

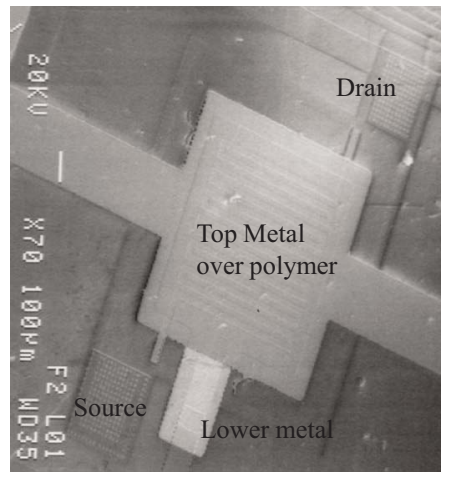

(d)

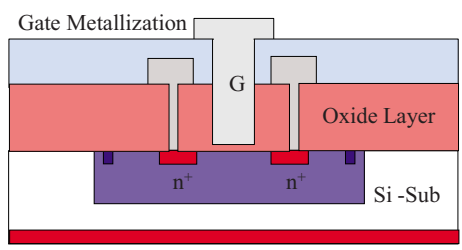

(i) n-MOS Device

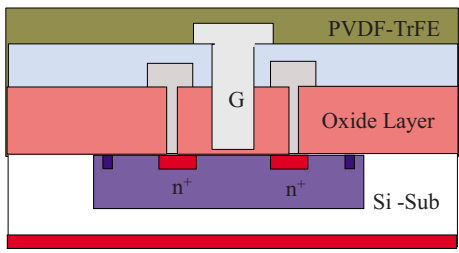

(ii) Polymer film Deposition

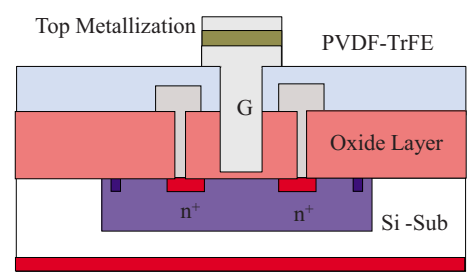

(iii) POSFET Device

(e)

FIG. 1. (Color online) POSFET touch sensing device implementation. (a) Structure and working of POSFET touch sensing device. (b) SEM image of POSFET cross section. (c) POSFET before polymer deposition. (d) SEM image of POSFET after polymer deposition. (e) Fabrication steps of POSFET touch sensing device.

\footnotetext{
a) Electronic mail: ravinder.dahiya @iit.it.

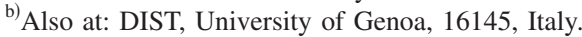



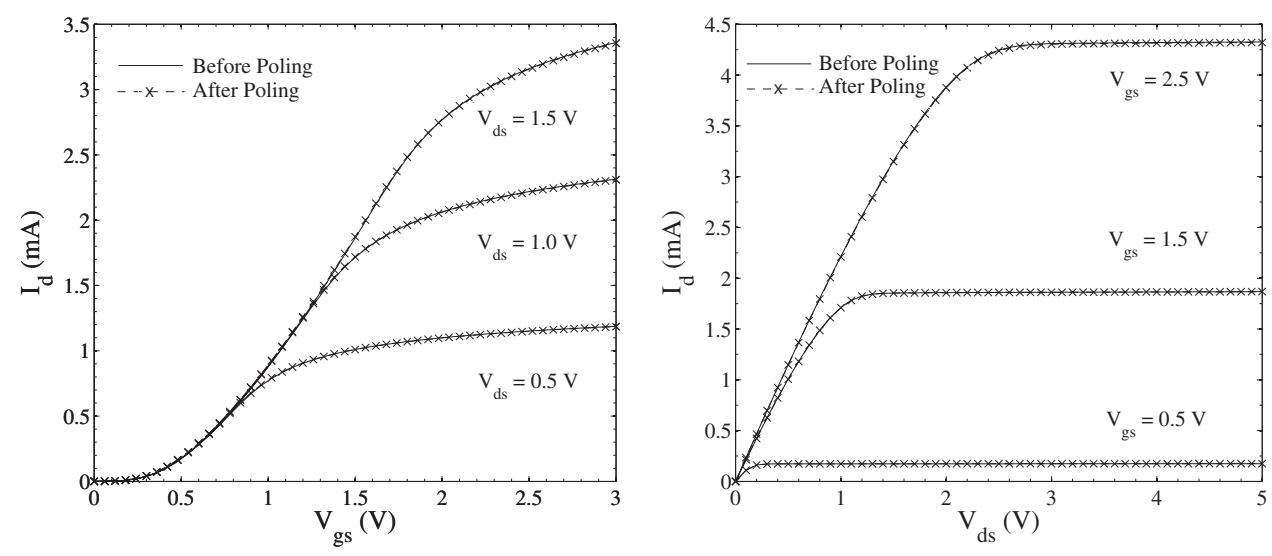

FIG. 2. Input characteristics (left) and output characteristics (right) before and after poling. developed for large area skin type of applications. ${ }^{3}$ However, best organics have a mobility of about $1 \mathrm{~cm}^{2} / \mathrm{V} \mathrm{s}$ versus $85 \mathrm{~cm}^{2} / \mathrm{V} \mathrm{s}$ for silicon based MOS devices, ${ }^{4}$ due to which their usage is limited to detecting and measuring slow varying contact forces only.

This work presents POSFET touch sensing devices, primarily developed for the fingertips of robotic hand. These devices are fabricated by spin coating thin $(\sim 2.5 \mu \mathrm{m})$ polyvinylidene fluoride trifluoroethylene $[\mathrm{P}(\mathrm{VDF}-\mathrm{TrFE})]$ film directly on the gate area of MOS transistor. The structure of POSFET device, shown in Figs. 1(a)-1(d), is similar to the metal-ferroelectric-metal-insulator-semiconductor type of ferroelectric random access memory (FeRAM). ${ }^{5}$ However, the thickness of ferroelectric film in FeRAM is usually much lower (100-200 nm) than that used in POSFET device. The fixed charges $\pm Q$, shown in Fig. 1(a), appear due to the remanent polarization $\left(P_{r}\right)$ of the piezoelectric polymer film and the charge neutrality condition. The charge carriers are thus accumulated at the surface of the semiconductor according to the polarization direction. For piezoelectric polymers in thickness mode, as in this work, the mechanical stress $\left(T_{3}\right)$, electric field $\left(E_{3}\right)$, and electric displacement $\left(D_{3}\right)$ are related as ${ }^{6}$

$$
D_{3}=d_{33} T_{3}+\epsilon_{33} E_{3},
$$

where $d_{33}$ and $\epsilon_{33}$ are, respectively, the piezoelectric and dielectric constants of piezoelectric polymer. Equation (1) shows that the electric displacement/charge and hence the polarization can be controlled by the electric field $\left(E_{3}\right)$ and the applied force $(F)$ or stress $\left(T_{3}\right)$. While former is used in FeRAM to switch the polarization state, the later is used in POSFET devices to modulate the charge in induced channel. Thus, the force variation is directly reflected into channel current of POSFET devices, which can be further processed by an electronic circuitry that may be present on the same chip. In this sense, POSFET device can also be compared with the mechanoreceptors in human skin-that not only sense the contact parameters, but also partially process the tactile data. ${ }^{7}$

In contrast with the conventional and extended gate approaches, 8,9 where transducers and conditioning electronics are separate entities connected through wires, the POSFET touch sensing devices allow sensing and processing at same site which will improve signal to noise ratio and the overall performance. A conformal electronic surface can be obtained by embedding such devices, as mechanically distinct and stiff subcircuit islands, in a soft and compliant poly- mer such as polydimethylsiloxane (PDMS) and interconnect the islands with flexible and stretchable metallization. ${ }^{10}$ Alternately, they can be developed on flexible electronic devices. $^{11}$

The fabrication steps for implementing POSFET touch sensing device are shown in Fig. 1(e). In first step, the MOS device is obtained using the $n$-MOS technological module of a nonstandard complimentary MOS (CMOS) technology, based on $4 \mu \mathrm{m} p$-well ion sensitive FET/CMOS process. ${ }^{12}$ A $\mathrm{Si}_{3} \mathrm{~N}_{4} / \mathrm{SiO}_{2}$ double layer is used as a gate dielectric. To match the tactile spatial acuity of human skin, POSFET devices are designed to have size of $1 \times 1 \mathrm{~mm}^{2}$ - fairly big when compared to the MOS devices obtained with present day technology. To obtain large transconductance, the $n$-MOS devices are designed to have $W=7500 \mu \mathrm{m}$ and $L$ $=12 \mu \mathrm{m}$. A $2.5 \mu \mathrm{m}$ thick PVDF-TrFE piezoelectric polymer film is then spin coated, at $3000 \mathrm{rpm}$ for $30 \mathrm{~s}$, from $10 \%$ concentrated solution. ${ }^{13}$ Further steps for in situ processing of polymer film include annealing at $120^{\circ} \mathrm{C}$ for $3 \mathrm{~h}$, followed by vacuum deposition of $200 \mathrm{~nm}$ metal $(\mathrm{Au} / \mathrm{Cr})$ on top of the polymer film and thereafter dry etching the polymer film. The $\mathrm{P}(\mathrm{VDF}-\mathrm{TrFE})$ film is then be polarized in situ by applying a voltage at the rate of $80-100 \mathrm{~V} / \mu \mathrm{m}(200-250 \mathrm{~V}$, in present case). With such a high voltage there is risk of damaging or altering the characteristics of underlying MOS device. To overcome this challenge, all metal layers and the substrate under the polymer film were grounded before ap-

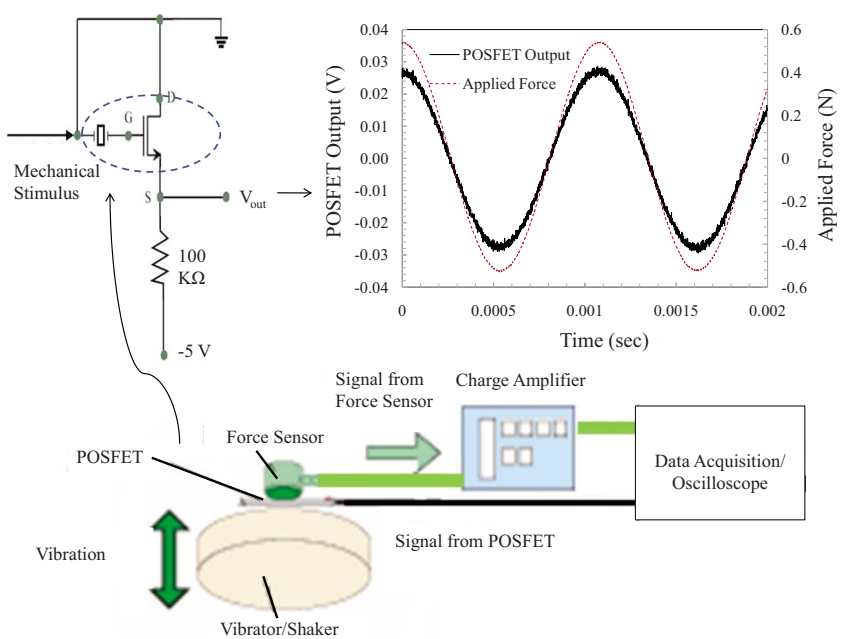

FIG. 3. (Color online) (top-left) POSFET connected in source-follower arrangement with floating gate (lower metal); (top-right) response of POSFET to a $1 \mathrm{~N}$ (peak-peak), $925 \mathrm{~Hz}$ sinusoidal force; and (bottom) experimental arrangement. 


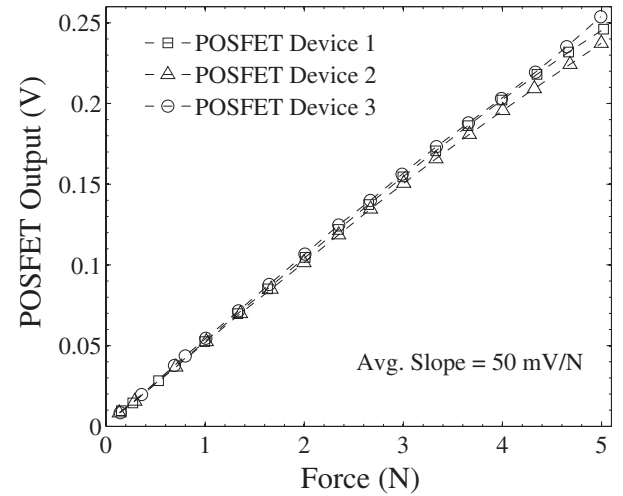

(a)

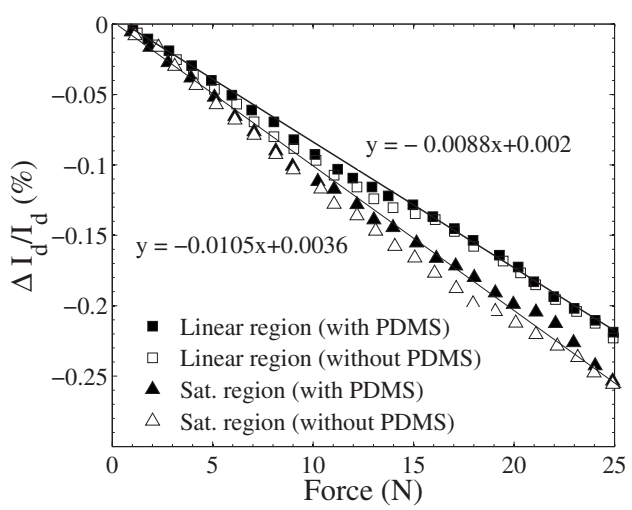

(b) plying the voltage across the polymer film. Poling was performed at $85{ }^{\circ} \mathrm{C}$, to reduce the voltage needed for polarization, and $200 \mathrm{~V}$ was finally used. Further care was taken by applying the voltage in four cumulative steps of $50 \mathrm{~V}$ each. ${ }^{13}$ The similar characteristics of POSFET touch sensing devices before and after the poling step, shown in Fig. 2, confirm that the steps taken to prevent device damage are sufficient.

The response of POSFET touch sensing device, to dynamic normal forces, is obtained by connecting it in a source-follower floating gate arrangement, as shown in Fig. 3. Source-follower configuration results in less than unity gain and hence POSFET devices can be tested for wide range of forces. Alternately, common source configuration can be used to have better force resolution. The experimental arrangement used to apply sinusoidal forces in the normal direction is also shown in Fig. 3. The POSFET device is pressed by vibration generator or shaker against the force sensor, which is equipped with a probe of dimensions 1.3 $\times 1.3 \mathrm{~mm}^{2}$. In this way the force applied by shaker is recorded by the force sensor and the output of POSFET is read on the oscilloscope. The output of a POSFET touch sensing device due to $925 \mathrm{~Hz}$ sinusoidal normal force applied on top of the device is shown in Fig. 3. When applying forces, the sensor is covered with a thin $300 \mu \mathrm{m}$ protective PDMS rubber. The response of three POSFET touch sensing devices, shown in Fig. 4(a), to a range of dynamic normal forces $(0.15-5 \mathrm{~N}$, sinusoidal, $20 \mathrm{~Hz})$ is linear with a slope of 50 $\mathrm{mV} / \mathrm{N}$. Considering various polymer and device parameters the expected response of POSFET device is approximately $78 \mathrm{mV} / \mathrm{N}$ - a value little higher than the observed response. This range of forces very well covers the range of forces $(0.15-0.9 \mathrm{~N})$ involved in normal manipulative tasks in humans. It should be noted that the drain current $\left(I_{d}\right)$ of MOS devices also changes with stress mainly as a result of mobility variations. ${ }^{14}$ In fact this piezoresistive property has been exploited to obtain MOS based pressure sensors. To understand the extent to which such effects contribute to the POSFET response, the $\mathrm{P}(\mathrm{VDF}-\mathrm{TrFE})$ film was removed and forces up to $25 \mathrm{~N}$ were applied with and without a thin PDMS film on the gate. For comparison the device was connected as in Fig. 3 and the gate voltage varied to obtain the response in linear and saturation regions. The response due to piezoresistive effect varies with biasing condition, ${ }^{15}$ as evident from plots in Fig. 4(b). With POSFET connected as in Fig. 3(a) $\Delta I_{d} / I_{d}$ of 0.01 is needed to obtain $50 \mathrm{mV} / \mathrm{N}$ output. The $\Delta I_{d} / I_{d}$ due to piezoresistive effect is about 0.0001 for $1 \mathrm{~N}$ i.e., $1 \%$ of $\Delta I_{d} / I_{d}$ needed to obtain $50 \mathrm{mV} / \mathrm{N}$ POSFET output. Hence the response of POSFET is mainly due to the piezoelectric behavior of PVDF-TrFE film.

In summary, the POSFET touch sensing devices have a linear response over wide range of forces. The response of POSFET is mainly due to piezoelectric behavior of $\mathrm{P}(\mathrm{VDF}-\mathrm{TrFE})$ film and other effects such as piezoresistive, that show up in presence of stress, contribute only to a minor extent. The POSFET devices as an integral sensor and electronic unit offer practical advantages such as reduction in number of wires which is essential for the target application, namely, fingertips of robotic hands.

This work was supported in part by European Commission project ROBOSKIN (Grant No. ICT-FP7-231500) and Italian MIUR project PRIN 2007 "Tactile Sensing System for Humanoid Robots using Piezo-polymer-FET devices."

${ }^{1}$ M. R. Cutkosky, R. D. Howe, and W. Provancher, Springer Handbook of Robotics (Springer, Berlin, Heidelberg, 2008).

${ }^{2}$ H. Takao, K. Sawada, and M. Ishida, IEEE Trans. Electron Devices 53, 1250 (2006)

${ }^{3}$ T. Someya, T. Sekitani, S. Iba, Y. Kato, H. Kawaguchi, and T. Sakurai, Proc. Natl. Acad. Sci. U.S.A. 101, 9966 (2004).

${ }^{4}$ D. H. Kim, J. H. Ahn, H. S. Kim, K. J. Lee, T. H. Kim, C. J. Yu, R. G. Nuzzo, and J. A. Rogers, IEEE Electron Device Lett. 29, 73 (2008).

${ }^{5}$ H. Ishiwara, Curr. Appl. Phys. 9, S2 (2009).

${ }^{6}$ R. S. Dahiya, M. Valle, and L. Lorenzelli, IEEE Trans. Ultrason. Ferroelectr. Freq. Control 56, 387 (2009).

${ }^{7}$ R. S. Johansson and I. Birznieks, Nat. Neurosci. 7, 170 (2004).

${ }^{8}$ R. G. Swartz and J. D. Plummer, IEEE Trans. Electron Devices 26, 1921 (1979).

${ }^{9}$ R. S. Dahiya, G. Metta, and M. Valle, Proceedings of the Fifth IEEE International Conference on Mechatronics (IEEE, New York, 2009), Vol. 1 , p. 1 .

${ }^{10}$ P. I. Hsu, M. Huang, Z. Xi, S. Wagner, Z. Suo, and J. C. Sturn, J. Appl. Phys. 95, 705 (2004)

${ }^{11}$ D. H. Kim, J. H. Ahn, W. M. Choi, H. S. Kim, T. H. Kim, J. Song, Y. Y. Huang, Z. Liu, C. Lu, and J. A. Rogers, Science 320, 507 (2008).

${ }^{12}$ S. Martinoia, N. Rosso, M. Grattarola, B. M. L. Lorenzelli, and M. Zen, Biosens. Bioelectron. 16, 1043 (2001).

${ }^{13}$ R. S. Dahiya, M. Valle, L. Lorenzelli, G. Metta, and S. Pedrotti, Proceedings of the IEEE Sensors 2008 (IEEE, New York, 2008), Vol. 1, p. 490.

${ }^{14}$ A. T. Bradley, R. C. Jaeger, J. C. Suhling, and K. J. O'Connor, IEEE Trans. Electron Devices 48, 2009 (2001).

${ }^{15}$ R. Vatedka, H. Takao, K. Sawada, and M. Ishida, Sens. Actuators, A 140, 89 (2007). 